\title{
A Case of hydropneumothorax
}

\section{Case report}

A 63-year-old female presents to the emergency department (ED) for increasing bilateral lower extremity edema over past two weeks.

A 63-year-old Caucasian female with past medical history of atrial fibrillation, pulmonary hypertension, end stage restrictive hypertrophic cardiomyopathy, severe tricuspid regurgitation, systemic hypertension, hypothyroidism, an implantable cardioverterdefibrillator (ICD), cardiovascular accident (CVA), cardiac cirrhosis, and jejunal cancer in remission presents to the ED for worsening bilateral extremity edema over the past two weeks. The patient's diuretics had been held due to increased creatinine levels, at which point her edema began to become worse (her legs increased in size). She was seen by her doctor's office and her diuretics were reinstated; however, her edema did not improve. She was then subsequently sent to the ER. Patient relates of increasing abdominal girth over the past two weeks as well. Patient has not been having any chest pressure, pain, dizziness. Does have mild increased breathlessness with ambulation. She has no cough no phlegm production. She denies increased salt consumption and she denies PND (paroxysmal nocturnal dyspnea) orthopnia.

Upon examination, it was found that she was mildly tachypneic and had JVD (Jugular vein distention). Sem noted in tricuspid region. Abdominal fullness with shifting dullness was also noted. A chest radiograph was preformed in emergency room revealed a moderate right pleural effusion. Her BNP was mildly elevated at 1900 (1-200), and her creatinine was 2.5 . She was subsequently admitted for lower extremity edema, abdominal ascites and right-sided pleural effusion related to her underlying cardiac disease. A CXR revealed minimal left effusion and moderate size right pleural effusion.

Upon admission she was started on IV diuretic therapy and was provided supplemental low flow oxygen. Imaging revealed fair amount of abdominal ascities and pleural effusion, for which interventional services were consulted. paracentesis.

She reported no shortness of breath, dyspnea upon exertion, chest pain, abdominal pain, fevers, chills, nausea, vomiting, constipation, dysuria, increased frequency, urgency, lightheadedness, palpitations, paroxysmal nocturnal dyspnea (PND), nor orthopnea.

The pleural effusion was transudative from cirrhosis, and we believe the hydropneumothorax portion of this process was due to prior scarring of her lung pleura, preventing re-expansion of the right lung. This chest $\mathrm{x}$-ray, as seen in Figure 1 shows cardiomegaly and right effusion.

On hospital day 4, patient had an ultrasound that reveals ascites. She underwent a paracentesis, and $2800 \mathrm{ml}$ of serous fluid was removed.

On hospital day 6, patient still had mild orthopnea thus underwent thoracic ultrasound. It was noted that she still had sizeable right pleural effusion. Thus, a therapeutic thoracentesis was performed and $1600 \mathrm{~mL}$ serous fluid was removed. A post-thoracentesis chest $\mathrm{x}$-ray seen in Figure 2 revealed a decreased right pleural effusion and trapped lung with right hydropneumothorax.
Volume 8 Issue 4 - 202I

\author{
Maaria Chaudhry,' Lidiya Didenko,' Kirill \\ Alekseyev,' Mohammad Umar Farooq, ${ }^{2}$ Bilal \\ Chaudhry ${ }^{2}$ \\ 'PAM Rehabilitation Hospital, USA \\ ${ }^{2}$ Christiana Care Health System, USA
}

Correspondence: Maaria Chaudhry, PAM Rehabilitation Hospital, USA, Tel 302672 5800, Email maariaaisha@gmail.com

Received: November 24, 2021 | Published: December 24, 2021

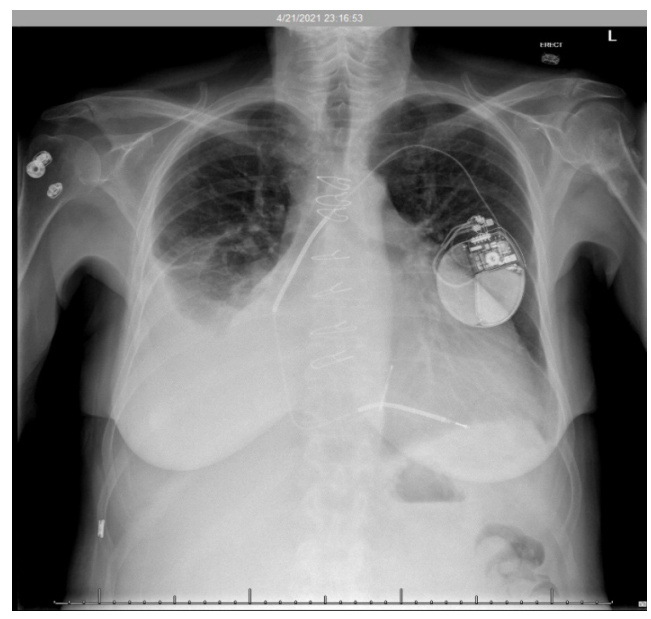

Figure I The radiograph depicts a right pleural effusion in addition to cardiomegaly.

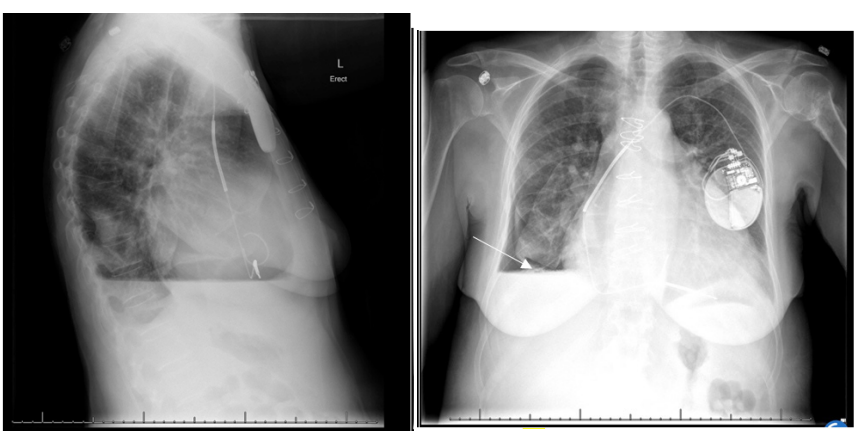

Figure 2 The radiograph depicts the air fluid level of the hydropneumothorax, as indicated by the white arrow. A minimal left pleural effusion in addition to cardiomegaly is also noted. 


\section{Discussion}

A hydropneumothorax occurs when free fluid and air enters the pleural space. While rare, it has been associated with malignancy, infection, chest trauma, rheumatologic diseases that affect lung parenchyma, following chest tube placement, and after a thoracentesis. It is the combination of a pneumothorax and hemothorax which may be seen in the setting of trauma but may also occur in the setting of non-traumatic situations. They may occur spontaneously or arise as a complication of cavitary and non-cavitary pulmonary metastases, and in rare occasions, they may also arise secondary to pulmonary manifestations of rheumatoid arthritis. ${ }^{1}$ Additionally, it may be associated with neurofibromatosis type 1 , hemophilia $\mathrm{A}$, and anticoagulation therapy. ${ }^{2}$

Differential diagnosis when considering a hydropneumothorax include hemothorax and pneumothorax. A hemothorax, describes a pleural effusion secondary to the accumulation of blood in the pleural cavity. Hemothorax should be considered in the setting of trauma, intrathoracic malignancies, anticoagulation medication and vascular rupture. ${ }^{3}$ A pneumothorax represents the presence of gas in the pleural space. It can occur without an underlying lung disorder, which is known as a primary spontaneous pneumothorax, or because of an underlying lung disorder, such a cystic lung diseases or parenchymal necrosis which is known as a secondary spontaneous pneumothorax. Additionally, a pneumothorax can also occur iatrogenic or secondary to trauma. ${ }^{4}$

Trapped lung is one of the outcomes of fibrinous or granulomatous pleuritis and is a cause of chronic, benign, unilateral pleural effusion. It is characterized by inability of the lung to expand and fill the thoracic cavity due to a restricting fibrous visceral pleural peel. The resulting chronic pleural space is fluid filled, and the persistence of the fluid is solely due to hydrostatic equilibrium. Historically recognized as a complication of therapeutic pneumothorax for treatment of tuberculosis, it is today most commonly a consequence of inadequately treated parapneumonic effusion, but it is also associated with cardiac surgery, chest trauma, and other inflammatory processes involving the pleura. The diagnosis requires documentation of chronicity and stability and the absence of an active inflammatory or malignant pleural process, bronchial obstruction, or severe underlying lung disease. Findings supporting the diagnosis are an initial negative pleural liquid pressure, increased pleural space elastance, and the demonstration of a pleural peel. Confirmation of the diagnosis requires successful surgical decortication, which is the only available therapy. ${ }^{5}$ In the asymptomatic patient, decortication is not indicated and observation is warranted. Unexpandable lung occurring as a consequence of active or remote pleural disease may present as a post-thoracentesis hydropneumothorax or an effusion that cannot be completely drained because of the development of anterior chest pain.
Chest radiographs in addition to thoracentesis provide definitive diagnosis when considering hydropneumothorax as the primary diagnosis. The majority of hemopneumothoraxes are treated with a reexpansion and drainage of the lung via prolonged tube thoracostomy for those who are poor candidates for surgery. For patients that are good candidates, such as those seen in the Characteristics of Trapped Lung, decortication proved successful. ${ }^{6}$ Additionally, Kohli described treatment of 66-year-old male with a left hydropneumothorax that was treated with thoracotomy, with complete decortication of the upper and lower left lobes and pleurectomy.

In this case, it was determined that a hydropneumothorax likely due to a bronchopleural fistula (BPF) formed from the patient's extensive necrotizing pneumonia. The breakdown of lung parenchyma allowed for a disruption in the bronchial wall and subsequent communication with the pleural space. On inspiration, air flows through the respiratory passages and enters the pleural space. The bronchial tear can produce a valve-like effect which allows air entry into the pleural space during inspiration but traps it during expiration. The diagnosis of hydropneumothorax is confirmed following a chest CT that reveals free air and fluid levels within the pleural space.

Prognosis for hydropneumothorax is good, so long as the underlying cause is benign source for the trapped lung, and granted that the rented lung heals quickly. She was cleared for discharge and instructed to return to the hospital if she experienced any shortness of breath, chest pain, shoulder/back pain, and/or lightheadedness. As for the hemopneumothorax, the thoracentesis was curative.

\section{References}

1. Weerakkody Y. Hemopneumothorax: Radiology Reference Article. Radiopaedia Blog. RSS. 2021

2. Weerakkody Y. Spontaenous hemopneumothorax: Radiology Reference Article. Radiopaedia Blog RSS. 2021.

3. Broderick SR. Hemothorax: Etiology, diagnosis, and management Thorac Surg Clin. 2013;23(1):89-96.

4. Swierzy M, Helmig M, Ismail M, et al Pneumothorax [Pneumothorax]. Zentralbl Chir. 2014;39(Suppl 1):S69-S86.

5. Doelken P. Clinical implications of unexpandable lung due to pleural disease. Am J Med Sci. 2008;335(1):21-25.

6. Huggins JT, Sahn SA, Heidecker J, et al. Characteristics of trapped lung: pleural fluid analysis, manometry, and air-contrast chest CT. Chest. 2007;131(1):206-213

7. Kohli E, Makkar H. Hydropneumothorax and trapped lung. J Emerg Med. 2015;48(2):211-212. 